\title{
Dyslipidemia in Patients with Chronic Kidney Disease (Dyslipidémie Chez Les Malades Rénaux Chroniques)
}

\section{Ramiandrisoa Lahatriniavo Ritchy ${ }^{\star, \dagger, 1}$, Ramilitiana Benja ${ }^{2}$, Razanakoto Naina Harinjara ${ }^{3}$, Randriamanantsoa Lova Narindra ${ }^{4}$, Randriamarotia Harilalaina Willy Franck $^{5}$}

\author{
${ }^{1}$ Service de Cardiologie, CHU Befelatanana, Antananarivo, Madagascar \\ ${ }^{2}$ Service de Néphrologie, CHU Befelatanana, Antananarivo, Madagascar \\ ${ }^{3}$ Service de Dermatologie, CHU Befelatanana, Antananarivo, Madagascar \\ ${ }^{4}$ Service de Réanimation Néphrologique, CHU Joseph Ravoahangy Andrianavalona, Antananarivo, Madagascat \\ ${ }^{5}$ Service de Néphrologie, CHU Befelatanana, Antananarivo, Madagascar
}

DOI: https://doi.org/10.15520/jcmro.v2i09.205

Accepted 26-09-2019; Received 13-09-2019; Publish Online 30-09-2019

Reviewed By: Dr. V.

Daniel

Department:

Reviewer/CMRO

\section{ABSTRACT}

Aims: Describe the lipid profile of patients with chronic kidney disease and to assess the role played by dyslipidemia in the kidney disease progression.

Patients and method: It was a retrospective descriptive and analytical study carried out at the Nephrology Unit of the University Hospital Befelatanana Antananarivo, from may 2008 to february 2015. We included in the study patients diagnosed as carriers of chronic kidney disease with abnormal lipid status. Patients who received henodialysis treatment were excluded.

Results: Ninety nine cases were selected, so as 48 men and 51 women. Their ages ranged from 18 to 87 years-old, with a mean of 50,47 years-old. Arterial hypertension was the most important comorbidity factor found, followed by age $(41,41 \%)$ and smoking (40,40\%). Patients with CKD stage 5 was the majority $(54,55 \%)$. Lipid profile was mainly compounded by Low HDL cholesterolemia (44,44\%). Hypertriglyceridemia was only found in $10,10 \%$ of cases. In univariate analysis, only a low HDL cholesterolemia was associated significantly with cardiovascular event $(\mathrm{p}=0,04)$. We found significant association between dyslipidemia and glomerular filtration rate in adjusted analysis with proteinuria $(\mathrm{p}<0,05)$.

Conclusion: Our study demonstrated that screening and treating abnormal cholesterolemia is necessary in patients with chronic kidney disease. Large-scale prospective studies, assessing the impact of treating dyslipidemia in kidney disease should be carried out.

(Résumé:

Buts: Décrire le profil lipidique des patients avec maladie rénale chronique et évaluer le rôle de la dyslipidémie dans la progression de la maladie rénale.

Méthode: II s'agissait d'une étude retrospective, descriptive et analytique réalisée dans le service de Néphrologie du Centre Hospitalier Universitaire Befelatanana Antananarivo du mois de mai 2008 à février 2015. Nous avons inclus dans l'étude les patients diagnostiqués comme porteurs de maladie rénale chronique avec un bilan lipidique anormal. Les patients ayant bénéficié d'un traitement de suppléance ont été exclus. Résultats: Quatre vingt dix neuf cas ont été retenus dont 48 hommes et 51 femmes. L'âge variait de 18 à 87 ans, avec une moyenne de 50,47 ans. L'hypertension artérielle était le facteur de comorbidité le plus important (79\%), suivi du tabagisme (40\%). Les patients au stade 5 de la maladie rénale chronique étaient majoritaires (54,55\%). Les anomalies lipidiques étaient prédominées par l'hypoHDL (44.44\%). L'hypertriglycéridémie n'a été retrouvée que dans $10.10 \%$ de cas. Ajustée à la protéinurie, les paramètres lipidiques à l'exception de la triglycéridémie étaient associés significativement à une baisse de la DFG $(\mathrm{p}<0,05)$.

Conclusion: Notre étude a démontré l'importance du traitement de l'anomalie de la cholestérolémie chez les malades rénaux chroniques. Des études prospectives sur l'impact du traitement de la dyslipidémie dans la maladie rénale devraient être effectuées dans une large population.) 


\section{INTRODUCTION:}

La maladie rénale chronique (MRC) se définit par la présence pendant plus de trois mois d'une diminution du débit de filtration glomérulaire (DFG), inférieure à 60 $\mathrm{ml} / \mathrm{mn} / 1,73 \mathrm{~m}^{2}$ et/ou la présence de marqueurs d'atteinte rénale [1] [2]. Les marqueurs d'atteinte rénale peuvent être des anomalies morphologiques (à l'échographie rénale), des anomalies histologiques (à la biopsie rénale) ou biologiques (leucocyturie, albuminurie, protéinurie, hématurie) [1].

Les dyslipidémies correspondent à une modification pathologique d'un ou de plusieurs paramètres des lipides sériques [3].

$\mathrm{Au}$ Canada, une étude multicentrique a montré une prévalence de la dyslipidémie chez les malades rénaux chroniques à $43,4 \%$ [4]. Une étude africaine a retrouvé une prévalence plus élevée à $70 \%$ [5]. A Madagascar, aucune donnée n'est disponible à ce sujet. Ainsi, nous proposons cette étude pour décrire le profil lipidique des patients avec maladie rénale chronique dans un premier temps et pour évaluer l'impact de la dyslipidémie dans l'apparition de maladies cardio- vasculaires et dans la progression de la maladie rénale dans un second temps.

\section{MÉTHODE:}

L'étude s'est déroulée dans le service de Néphrologie du CHU Befelatanana d'Antananarivo (centre national de référence). Il s'agit d'une étude retrospective descriptive et analytique sur une période de 06 ans et 10 mois (mai 2008 à février 2015). Nous avons inclus dans l'étude les patients admis dans le service avec bilan lipidique anormal, diagnostiqués comme porteur de MRC. Les patients ayant bénéficié d'un traitement de suppléance ont été exclus.

Le diagnostic de dyslipidémie était posé devant: un taux de cholestérol total supérieur à 2,20 $\mathrm{g} / \mathrm{L}(5,7 \mathrm{mmol} / \mathrm{L})$ ou un taux de triglycérides supérieur à $1,50 \mathrm{~g} / \mathrm{L}(1,7 \mathrm{mmol} / \mathrm{L})$ ou un taux de HDL- Cholestérol inférieur à $0,45 \mathrm{~g} / \mathrm{L}$ chez l'homme et inférieur à $0,55 \mathrm{~g} / \mathrm{L}$ chez la femme $[6,7]$.

La maladie rénale chronique était définie par la persistance de l'un des signes d'atteinte rénale suivants, pendant plus de trois mois sur 2 examens consécutifs [8,1]: DFG $<60$ $\mathrm{mLmn} / 1,73 \mathrm{~m}^{2}$; protéinurie ou albuminurie positive, hématurie : GR $<10000 / \mathrm{mL}$ ou $<10 / \mathrm{mm} 3$, leucocyturie : GB $>10 / \mathrm{mm}^{3}$ ou $>10000 / \mathrm{mL}$ en absence d'infections, anomalie morphologique à l'échographie rénale : reins de petite taille, gros reins polykystiques, asymétrie de taille, contours bosselés, avec dédifférenciation cortico-médullaire, kystes.

Les données ont été traitées à l'aide du logiciel Epi info7.1.3.3 ${ }^{\circledast}$ et Microsoft Excel 2010 ${ }^{\circledast}$.

\section{RÉSULTATS:}

3.1. Aspects épidémiologiques et cliniques: Nous avons enregistré 140 patients présentant une MRC dont 99

* Corresponding author.

† Email: ritchyram@yahoo.com soit 70,7\% avaient une dyslipidémie associée. L'âge moyen de nos patients inclus était de 50,47 ans, avec un minimum de 18 et un maximum de 87 ans. Le genre féminin était prédominant et représentait $51,5 \%$ de notre population d'étude avec un sex ratio de 0,94 .

Nous avons enregistré 140 patients présentant une MRC dont 99 soit 70,7\% avaient une dyslipidémie associée. L'âge moyen de nos patients inclus était de 50,47 ans, avec un minimum de 18 et un maximum de 87 ans. Le genre féminin était prédominant et représentait $51,5 \%$ de notre population d'étude avec un sex ratio de 0,94 .

Outre la dyslipidémie, nous avons réparti les patients selon le nombre et le type des autres facteurs de risque cardio-vasculaire. Ainsi, la majorité des patients $(73,7 \%)$ avait plus de 2 facteurs de risque cardio-vasculaire en plus de la dyslipidémie.

L'hypertension artérielle (HTA) était le facteur de risque prédominant, retrouvée chez 79 patients soit $79,80 \%$, suivie du tabagisme (40,40\%). Le diabète était retrouvé dans $17,17 \%$ des cas Table 1 (tableau I).

Concernant les chiffres tensionnels, la moyenne de la tension artérielle était de 160,08 $\pm 31,80 \mathrm{mmHg}$ pour la systolique et de 95,71 $\pm 20,70 \mathrm{mmHg}$ pour la diastolique. Quarante cinq patients étaient hypertendus grade III $(45,45 \%)$. Dix neuf patients $(19,19 \%)$ avaient une tension artérielle normale Table 1 (tableau I).

3.2. Données biologiques: La créatininémie moyenne était de $686,26 \mu \mathrm{mol} / \mathrm{L}$ avec un minimum de 107 et un maximum de $2424 \mu \mathrm{mol} / \mathrm{L}$. Les patients avec une créatininémie entre 100 et $500 \mu \mathrm{mol} / \mathrm{L}$ étaient de 54,55\% Table 1 (tableau I). Le DFG était estimé à partir de la formule de MDRD simplifiée. La moyenne était de 19,11 mL/min, variant entre 2 et $58 \mathrm{~mL} / \mathrm{min}$. Cinquante trois patients $(53,5 \%)$ présentaient une insuffisance rénale chronique stade 5 avec un DFG $<15 \mathrm{~mL} / \mathrm{min}$ Table 1 (tableau I). La protéinurie de $24 \mathrm{~h}$ de nos patients variait de 0 à $12,79 \mathrm{~g}$ avec une moyenne de $1,66 \mathrm{~g}$. La protéinurie entre 0 à $1 \mathrm{~g} / 24 \mathrm{~h}$ avait une fréquence de $56,57 \%$.

L'uricémie variait de 281 à $1210 \mu \mathrm{mol} / \mathrm{L}$ avec une moyenne de $601,45 \pm 202,51 \mu \mathrm{mol} / \mathrm{L}$. Trente huit patients avaient une hyperuricémie $(38,4 \%)$.

Le type de dyslipidémie prédominant était le High density lipoprotein cholestérol (HDL c) bas, retrouvé chez 44 patients : soit 44,4\% de notre population d'étude Table 1 (tableau I).

La dyslipidémie survient indépendamment de l'âge ( $p$ $>0,05$ pour chacun des types de dyslipidémie) et du sexe ( $p>0,05$ pour chacun des types de dyslipidémie). Nous n'avons pas trouvé de relation significative entre le DFG et la cholestérolémie totale élevée $(p=0,46)$, ou l'hypertriglycéridémie $(\mathrm{p}=0,29)$, ou le HDL c bas ( $\mathrm{p}=$ 0,37), ou le Low density lipopretein cholesterol (LDL c élevé) $(\mathrm{p}=0,39)$. Après ajustement à la protéinurie, l'association devenait significative pour la cholestérolémie totale $(\mathrm{p}<$ $0,001)$, le HDL c bas $(p=0,01)$ et le LDL c élevé $(p<0,001)$; l'association entre le DFG et la triglycéridémie restait non significative $(\mathrm{p}=0,05)$.

3.3. Données thérapeutiques: Quarante neuf patients $(49,5 \%)$ ont été mis sous statine : l'atorvastatine 
était utilisée chez 47 patients $(47,47 \%)$; les 2 autres patients étaient sous simvastatine. Les patients qui n'avaient pas reçu d'hypocholestéroliémiant $(57,1 \%)$ avaient une dyslipidémie à type de HDL c bas Table 2 (tableau II). Nous avons trouvé une relation statistiquement significative entre le DFG et l'utilisation des statines $(p=0,008)$.

Table 1. Répartition des patients selon les données clin-iques et biologiques

\begin{tabular}{|c|c|c|}
\hline & $\begin{array}{l}\text { Ef- } \\
\text { fectif }\end{array}$ & $\begin{array}{l}\text { Pour- } \\
\text { centage }\end{array}$ \\
\hline \multicolumn{3}{|l|}{ Nombre de FDRCV cumulés } \\
\hline$<3$ & 26 & 26,3 \\
\hline$\geq 3$ & 73 & 73,7 \\
\hline \multicolumn{3}{|l|}{ FDRCV présentés } \\
\hline HTA & 79 & 79,8 \\
\hline Tabagisme & 40 & 40,4 \\
\hline Hérédité cardiovasculaire & 34 & 34,3 \\
\hline Ménopause & 7 & 7,1 \\
\hline Diabète & 17 & 17,2 \\
\hline Obésité & 4 & 4,1 \\
\hline Sédentarité & 3 & 3,0 \\
\hline HVG & 20 & 20,2 \\
\hline \multicolumn{3}{|l|}{ Tension artérielle (mmHg) } \\
\hline Normale (120-129 / 80-84) & 12 & 12,1 \\
\hline Normale haute (130-139 / 85-89) & 7 & 7,1 \\
\hline HTA grade 1 (140-159 / 90-99) & 12 & 12,1 \\
\hline HTA grade 2 (160-179 / 100-109) & 23 & 23,2 \\
\hline HTA grade $3(\geq 180 / \geq 110)$ & 45 & 45,4 \\
\hline \multicolumn{3}{|l|}{ Créatininémie $(\mu \mathrm{mol} / \mathrm{L})$} \\
\hline 100 à 500 & 54 & 54,5 \\
\hline 501 à 1000 & 21 & 21,2 \\
\hline$>1000$ & 24 & 24,2 \\
\hline \multicolumn{3}{|l|}{ DFG $(\mathrm{mL} / \mathrm{min}) /$ Stade MRC } \\
\hline$<15 /$ Stade 5 & 53 & 53,5 \\
\hline 15 à $29 /$ Stade 4 & 23 & 23,2 \\
\hline 30 à $44 /$ Stade $3 \mathrm{~B}$ & 9 & 9,1 \\
\hline 45 à 60 / Stade $3 \mathrm{~A}$ & 14 & 14,1 \\
\hline Type de dyslipidémie & 36 & 36,4 \\
\hline LDL c élevé & 44 & 44,4 \\
\hline HDL c bas & 10 & 10,1 \\
\hline Hypertriglycéridémie & 9 & 9,1 \\
\hline Dyslipidémie mixte & & \\
\hline
\end{tabular}

DFG : débit de filtration glomérulaire ; FDRCV : facteurs de risque cardio-vasculaire ; HTA : hypertension artérielle ; HVG : hypertrophie ventriculaire gauche ; HDL c : high density lipoprotein cholesterol ; LDL c : low density lipoprotein cholesterol ; $\mathrm{MRC}$ : maladie rénale chronique

Table 2. Répartition des patients selon le traitement et le type de dyslipidémie

\begin{tabular}{lll}
\hline $\begin{array}{l}\text { Type de } \\
\text { dyslipidémie }\end{array}$ & $\begin{array}{l}\text { Absence de statine } \\
\mathrm{n}(\%)\end{array}$ & $\begin{array}{l}\text { Présence de statine } \\
\mathrm{n}(\%)\end{array}$ \\
& $\mathrm{n}=49$ & $\mathrm{n}=49$ \\
& $12(24,5)$ & $24(48,9)$ \\
LDL c élevé & $28(57,1)$ & $16(32,6)$ \\
$\begin{array}{l}\text { HDL c bas } \\
\text { Hypertrigly- }\end{array}$ & $7(14,3)$ & $3(6,1)$ \\
$\begin{array}{l}\text { céridémie } \\
\text { Dyslipidémie } \\
\text { mixte }\end{array}$ & $2(4,1)$ & $6(12,2)$ \\
\hline
\end{tabular}

LDL c : low density lipoprotein cholesterol; HDL c : high density lipoprotein cholesterol

\section{DISCUSSION:}

4.1. Données épidémiologiques et cliniques: La fréquence de la dyslipidémie sur MRC était de 70,7\% dans notre étude, qui était similaire à la littérature. En effet, Kohlhagen $\mathrm{J}$ et al et Kaba ML et al [8] avaient retrouvé respectivement une fréquence de $70,1 \%$ sur 150 patients et $70,2 \%$ sur 44 patients présentant une $\operatorname{MRC}[5,9]$.

L'âge moyen était de 50,5 ans dans notre étude, comparable à celui retrouvé par Chawla $\mathrm{V}$ et al $(52,4$ ans) et Chen $\mathrm{SC}$ et al $(60,6$ ans) $[10,11]$. La dyslipidémie est rare avant ces âges, en dehors des formes familiales.

Soixante treize patients $(73,3 \%)$ avait cumulé plus de 2 facteurs de risque cardio-vasculaire associés à la dyslipidémie. Parikh NI et al [12] ont aussi retrouvé sur une étude concernant les facteurs de risque cardio-vasculaire dans la MRC que $73 \%$ des patients ont plus de 2 facteurs de risque, renforçant le fait que le risque cardio-vasculaire global est très élevé au cours de la MRC.

Outre la dyslipidémie, l'HTA était le facteur de risque cardio-vasculaire le plus fréquent $(79,8 \%$ des cas), qui était aussi le premier facteur de risque dans les séries de Kaba ML et al $(70,7 \%)$ et Chen SC et al $(66,6 \%)[5,11]$. L'HTA pourrait être à la fois la cause et la conséquence de la MRC $[11,13]$.

La valeur moyenne de la tension artérielle était de 160,1 $\mathrm{mmHg}$ pour la systolique et $95,7 \mathrm{mmHg}$ pour la diastolique, largement supérieure à celles retrouvées par Rahman et al (131,5 $\mathrm{mmHg}$ de systole et $74,9 \mathrm{mmHg}$ de diastole) [11] et Chawla V $(134,4 \mathrm{mmHg}$ de systole et $72,5 \mathrm{mmHg}$ de diastole [14]. Cette différence dans notre étude est expliquée probablement par une méconnaissance de l'HTA ou une mauvaise observance thérapeutique avant l'hospitalisation mais aussi un mauvais contrôle tensionnel lorsqu'elle est connue, de cause multifactorielle comme le coût des médicaments dans un pays à revenu faible.

4.2. Données biologiques: Le DFG variait de 2 à $58 \mathrm{~mL} / \mathrm{min}$ dans notre étude avec une moyenne de 19,11 $\mathrm{mL} / \mathrm{min}$. Chawla $\mathrm{V}$ et al et Rahman et al avaient retrouvé respectivement un DFG moyen de $32,8 \mathrm{~mL} / \mathrm{min}$ [10] et de $45,1 \mathrm{~mL} / \mathrm{min}$ [15] . Cinquante trois patients (53,53\%) étaient au stade 5 de la MRC contre seulement $31,1 \%$ pour Chen SC et al [11].

C'est le reflet du retard diagnostique de la MRC à Madagascar mais aussi de la dyslipidémie. L'étude ARIC (The Atherosclerosis Risk in Communitites), concernant la dyslipidémie et le risque de développement de dysfonction rénale rapporte qu'une élévation de la triglycéridémie ou une baisse de l'HDL-cholestérolémie s'accompagne d'une élévation de la créatininémie de $0,4 \mathrm{mg} / \mathrm{dL}$ ou plus [16].

Au cours de l'analyse univariée, nous n'avons pas trouvé de corrélation significative entre le DFG et chacun des valeurs respectives de la cholestérolémie totale, de la LDLc, de la HDL-cholestérolémie et de la triglycéridémie. Mais ajustée à la protéinurie, l'association devenait significative pour la cholestérolémie totale élevée, la LDL c élevée et le HDL c basse. L'association avec la triglycéridémie était inchangée. Ceci peut expliquer par le fait que la prévalence de la dyslipidémie au cours de la MRC varie à la fois en 
fonction de la sévérité de la maladie et aussi du degré de la protéinurie [17, 18].

4.3. Données thérapeutiques: Dans notre étude, $49,49 \%$ des patients ont été mis sous statines contre 39,6\% et $33,2 \%$ respectivement pour celui de Chen SC et al et Rahman et al[35, 36]. Malgré l'intérêt de la statine dans la MRC, des fibrates ont été utilisés dans $8,9 \%$ des cas pour Chen SC et dans 11,2\% des cas pour Rahman [19-21]. Les patients qui n'ont pas reçus de statine dans notre étude étaient surtout des patients avec une hypo-HDL-cholestérolémie (57,14\%), même si ce type de dyslipidémie devrait aussi bénéficier d'un traitement par statine.

\section{CONCLUSION:}

La dyslipidémie est très fréquente dans notre étude, comme dans la littérature. Les patients ont cumulés plus de 2 facteurs de risque cardio-vasculaire en plus de la dyslipidémie, reflétant le risque cardio-vasculaire global très élevé de ces patients.

Ajustée à la protéinurie, la cholestérolémie totale élevée, la LDL c élevée et le HDL bas ont une relation négative par rapport au DFG.

Une étude de suivi dans une population beaucoup plus large est utile pour mieux étudier l'impact de la dyslipidémie sur la progression de la MRC dans la population malgache.

Conflits d'intérêts: Les auteurs ne déclarent aucun conflit d'intérêt.

Contributions des auteurs: Tous les auteurs ont contribué à la conduite de ce travail. Tous les auteurs déclarent également avoir lu et approuvé la version finale du manuscrit.

\section{REFERENCES}

1. Agence nationale d'accréditation et d'évaluation en santé. Diagnostic de l'insuffisance rénale chronique chez l'adulte. Recommandations pour la pratique clinique. Paris: ANAES; 2002.

2. Alani H, Tamimi A, Tamimi N. Cardiovascular co-morbidity in chronic kidney disease: Current knowledge and future research needs. World J Nephrol. 2014; 3(4): 156-68.

3. Faure E, Labreze L. Les dyslipidémies. 2002 Juin. Consultable à l'URL:http://www.caducee.net/DossierSpecialises/endocrinologie/ dyslipidemie.asp

4. Tonelli M, Bohm C, Pandeya S, Gill J, Levin A, Kiberd BA. Cardiac risk factors and the use of cardioprotective medications in patients with chronic renal insuffiency. Am J Kidney Dis. 2001; 37 (3): 484-9. 5. Kaba ML, Diakite M, Bah AO, Sylla IS, Cherif I, Tolino A et al. Profil lipidique des urémiques à l'hôpital de Donka à Conakry (Guinée). Med Afr Noire. 2010; 57 (5): 255-8

6. Riesen WF, Virgini V, Vogt B, Rotondi N. Nouvelles guidelines européennes pour la dyslipidémie. Rev Med Suisse. 2012 Mars ; 8: 525-30.

7. Agence nationale d'accréditation et d'évaluation en santé. Modalités de dépistage et diagnostic des dyslipidémies en prévention primaire. Paris : Anaes ; 2000

8. Haute Autorité de Santé. Maladie rénale chronique de 1'adulte. Guide du parcours de soins. Saint-Denis La Plaine : HAS ; 2012.

9. Kohlhagen J, Kelly JJ. Atherosclerotic risk factors and vascular disease in chronic impairment. Nephrology. 2002; 7 (Suppl): A1-A40
10. Chawla V, Greene T, Beck G, Kusek JW, Collins AJ, Sarnak MJ et al. Hyperlipidemia and long-term outcomes in nondiabetic chronic kidney disease. Clin J Am Soc Nephrol. 2010; 5: 1582-7.

11. Chen SC, Hung CC, Kuo MC, Lee JJ, Chiu YW, Chang JM et al. Association of dyslipidemia with renal outcomes in chronic kidney disease. PLoS ONE 8(2): e55643. doi:10.1371/journal.pone.0055643 12. Parikh NI, Hwang SJ, Larson MG, Meigs JB, Levy D, Fox CS. Cardiovascular disease risk factors in chronic kidney disease. Arch Intern Med. 2006; 25: 1884- 91.

13. Manttari M, Tiula E, Alikoski T, Manninen V. Effects of Hypertension and dyslipidemia on the decline in renal function. Hypertension. $1995 ; 26: 670-5$.

14. Youmbissi TJ, Djoumessi S, Simo Moyo J, Nouedoui C, Tchemy C, Zam Ngono EC. Profil lipidique général d'un groupe de malades insuffisants rénaux chroniques Camerounais. Med Afr Noire. 1994; 41 (1).

15. Rahman M, Yang W, Akkena S, Alper A, Anderson AH, Appel LJ et al. Relation of serum lipids and lipoproteins with progression of CKD: The CRIC study. Clin J Am Soc Nephrol. 2014; 9: 1190-8. 16. Muntner P, Coresh J, Smith C, Eckfeldt J, Klag MJ. Plasma lipids and risk of developing renal dysfunction: The atherosclerosis risk in communities study. Kidney Int. 2000; 58: 293-301.

17. Szumilak D, Nguyen TK, Touam M, Jungers P, Lacour B, Massy ZA. Lipides et risque cardiovasculaire au cours de l'insuffisance rénale chronique. Nutr Clin Métabol. 1999; 13: 187-90.

18. Panagiotis T, Vlagopoulos, Sarnak MJ. Traditional and Nontraditional cardiovascular risk factors in chronic kidney disease. Med Clin Am. 2005; 89: 587-611.

19. Özsoy R, Leuven SI, Kastelein JJP, Arisz L, Koopman MG. The dyslipidemia of chronic renal disease. Curr Opin Lipodol. 2006; 17 (6): 659-66.

20. Kimura K, Shimano H, Yokote K, Urashima M, Teramoto T. Effect of pitavastatin (LIVALO tablet) on the estimate glomerular filatration rate (eGFR) in hypercholesterolemic patients with chronic kidney disease. Sub-analysis of LIVALO Effectiveness and safety (LIVES) study. J Atheroscl Thromb. 2010; 17 (6): 601-9.

21. Nakamura H, Mizuno K, Ohashi Y, Yoshida T, Hirao K, Uchida Y et al. Pravastatin and cardiovascular risk in moderate chronic kidney disease. Atherosclerosis. 2009; 206 (2): 512-7. 